\title{
THE GAME QUANTIFIER ${ }^{1}$
}

\author{
YIANNIS N. MOSCHOVAKIS
}

ABSTRACT. For structures that satisfy certain mild definability conditions we show the following result: $A$ relation $R\left(x_{1}, \cdots, x_{n}\right)$ has a positive first order inductive definition iff for some first order $Q\left(y, x_{1}, \cdots, x_{n}\right)$,

$$
\begin{aligned}
R\left(x_{1}, \cdots, x_{n}\right) & \\
& \Leftrightarrow\left(\forall y_{1} \exists y_{2} \forall y_{3} \exists y_{4} \cdots\right)(\exists k) Q\left(\left\langle y_{1}, \cdots, y_{k}\right\rangle, x_{1}, \cdots, x_{n}\right) .
\end{aligned}
$$

This yields easily some of the basic results about inductive definability, e.g., the existence of a universal inductively definable set.

The theory of hyperarithmetical relations on the integers has been generalized to arbitrary first order structures in [5], [6]. Most of the known characterizations of hyperarithmeticalness hold in the abstract; however the basic Suslin-Kleene theorem holds only for countable structures (Barwise-Gandy-Moschovakis [1], Moschovakis [7]) while in general the hyperprojective subsets of a structure are a proper subclass of the $\Delta_{1}^{1}$ sets. Because of this there has been no simple syntactical characterization of the hyperprojective sets. We give here such a characterization using infinite strings of alternating quantifiers in the manner of Keisler [3].

According to the normal form theorem (Kleene [4]), each $\Pi_{1}^{1}$ relation on $\omega$ is obtained by a single application of the Suslin quantifier (the dual of the classical operation $\mathcal{A}$ ) to an arithmetical (or even recursive) relation:

$$
\begin{aligned}
R\left(x_{1}, \cdots, x_{n}\right) & \Leftrightarrow(S y) Q\left(y, x_{1}, \cdots, x_{n}\right) \\
\Leftrightarrow & \left(\forall y_{1} \forall y_{2} \forall y_{3} \forall y_{4} \cdots\right)(\exists k) Q\left(\left\langle y_{1}, \cdots, y_{k}\right\rangle, x_{1}, \cdots, x_{n}\right) .
\end{aligned}
$$

It is rather amusing and easy to prove that we get another normal form for $\Pi_{1}^{1}$ relations if we use the game quantifier instead, i.e. if we change every other $\forall$ to $\exists$ :

$$
\begin{aligned}
R\left(x_{1}, \cdots, x_{n}\right) & \Leftrightarrow(\mathcal{G}) Q\left(y, x_{1}, \cdots, x_{n}\right) \\
& \Leftrightarrow\left(\forall y_{1} \exists y_{2} \forall y_{3} \exists y_{4} \cdots\right)(\exists k) Q\left(\left\langle y_{1}, \cdots, y_{k}\right\rangle, x_{1}, \cdots, x_{n}\right) .
\end{aligned}
$$

Received by the editors March 23, 1970.

AMS 1969 subject classifications. Primary 0277; Secondary 0440.

Key words and phrases. Inductive definability, abstract hyperarithmetic sets, hyperprojective, infinite alternating quantifier strings.

${ }_{1}^{1}$ The preparation of this paper was sponsored in part by NSF Grant GP-8827. 
(Of course we must give a correct interpretation to the infinite alternating string in (2); see §1.) Now (1) and (2) make sense not only on the integers but over any structure in which we can define codings for tuples, in particular, any acceptable structure $\mathfrak{A}$ in the sense of Moschovakis [7]. For some of these structures the Suslin quantifier preserves first order definability, because we can code infinite sequences by single elements of the structure. This is in fact true for the standard model of second order number theory with domain $\omega \cup^{\omega} \omega$ which provided the initial motivation for studying hyperprojective relations. The game quantifier however goes beyond first order definability and gives a normal form for the abstract analog of $\boldsymbol{\Pi}_{1}^{1}$.

Definition. Let $\mathfrak{A}=\left\langle A, R_{1}, \cdots, R_{l}, f_{1}, \cdots, f_{m}\right\rangle$ be acceptable. A relation $R\left(x_{1}, \cdots, x_{n}\right)$ on $A$ is $\mathbf{S}_{1}$ if for some (first order) definable (with parameters from $A$ ) relation $Q\left(y, x_{1}, \cdots, x_{n}\right)$,

$$
R\left(x_{1}, \cdots, x_{n}\right) \Leftrightarrow(\mathcal{S} y) Q\left(y, x_{1}, \cdots, x_{n}\right) .
$$

MaIN Result. Let $\mathfrak{A}$ be acceptable. The $\mathfrak{S}_{1}$ relations on A are precisely the inductively definable (or semihyperprojective) relations on $A$.

One can easily piece together a proof of this result using the techniques developed in [5] and in particular the game-theoretic characterization of semihyperprojectivity given in $\S 19$ of that paper. The point of this note is that we give a direct and elementary proof of the normal form (3) for inductively definable relations, a proof that does not depend on the complicated calculus of hyperprojective functions developed in [5]. In $\S 3$ we indicate how this can be used to give fairly direct proofs of the basic theorems about inductive definability, e.g. the existence of a universal inductively definable set.

To save space, we shall assume the notation and terminology established in $\S 1-\S 3$ of [7]. The result of that paper however is not essential for understanding the main argument in this note.

1. Elementary transformations. The operator $S$ on an acceptable structure can be immediately understood as quantification over all countable sequences in $A$ :

$$
\left(\mathcal{S}_{y}\right) Q(y) \Leftrightarrow\left(\forall f \in{ }^{\omega} A\right)(\exists k) Q(\langle f(1), \cdots, f(k)\rangle) .
$$

To interpret $(\mathcal{S} y) Q(y)$, consider the infinite two-player game where the first player (call him $\forall$ ) chooses $y_{1}$, the second player (call him $\exists$ ) chooses $y_{2}$, then $\forall$ chooses $y_{3}$, then $\exists$ chooses $y_{4}$, etc. ad infinitum, and $\exists$ wins iff for some $k, Q\left(\left\langle y_{1}, \cdots, y_{k}\right\rangle\right)$. Put

$$
(\mathcal{S}) Q(y) \Leftrightarrow \exists \text { has a winning strategy. }
$$

Strategies are functions on finite tuples from $A$ to $A$. If $\sigma * \tau$ is the play 
resulting when $\forall$ 's strategy $\sigma$ is pitted against $\exists^{\prime}$ 's strategy $\tau$; we can rewrite (4) as

$$
(\mathcal{G}) Q(y) \Leftrightarrow(\exists \tau)(\forall \sigma)(\exists k) Q(\langle\sigma * \tau(1), \cdots, \sigma * \tau(k)\rangle) .
$$

This game is open in the terminology of Gale-Stewart [2] and by the basic result of that paper it is determined, i.e. either $\forall$ or $\exists$ must have a winning strategy. Thus we can also write

$$
(\mathcal{G} y) Q(y) \Leftrightarrow(\forall \sigma)(\exists \tau)(\exists k) Q(\langle\sigma * \tau(1), \cdots, \sigma * \tau(k)\rangle) .
$$

Another easy consequence of the Gale-Stewart theorem is that

$$
\neg(\mathcal{S y}) Q(y) \Leftrightarrow\left(\mathcal{G}^{\smile} y\right) \neg Q(y),
$$

where $\mathcal{G}^{\smile}$ is the dual quantifier defined by

$$
\left(\mathcal{G}^{\smile} y\right) Q(y) \Leftrightarrow\left(\exists y_{1} \forall y_{2} \exists y_{3} \forall y_{4} \cdots\right)(\forall k) Q\left(\left\langle y_{1}, \cdots, y_{k}\right\rangle\right) .
$$

It is easy to verify the following equivalences. (Recall from [7] that if Seq $(x)$, then $K(x)$ is the length of the sequence that $x$ codes and $(x)_{i}$ ( $1 \leqq i \leqq K(x))$ is the $i$ th term of that sequence.)

$$
\begin{gathered}
(\mathcal{G} x) P(x) \&(\mathcal{G} y) Q(y) \Leftrightarrow(\mathcal{G} x)(\mathcal{G} y)[P(x) \& Q(y)] . \\
(\mathcal{G} x) P(x) \vee(\mathcal{G} y) Q(y) \Leftrightarrow(\mathcal{G} x)(\mathfrak{G} y)[P(x) \vee Q(y)] . \\
(\forall x) P(x) \Leftrightarrow(\mathcal{G} x)\left[\text { Seq }(x) \& K(x) \geqq 1 \& P\left((x)_{1}\right)\right] . \\
(\exists x) P(x) \Leftrightarrow(\mathcal{G} x)\left[\text { Seq }(x) \& K(x) \geqq 2 \& P\left((x)_{2}\right)\right] . \\
(\mathcal{S} x) P(x) \Leftrightarrow(\mathcal{G} x) Q(x),
\end{gathered}
$$

where

$$
\begin{gathered}
Q(x) \Leftrightarrow \text { Seq }(x) \& K(x) \text { is odd \& } P\left(\left\langle(x)_{1},(x)_{3}, \cdots,(x)_{K(x)}\right\rangle\right) . \\
(\mathcal{G} x)(\mathcal{G} y) P(x, y) \Leftrightarrow(\mathcal{G}) Q(z),
\end{gathered}
$$

where

$$
\begin{array}{r}
Q(z) \Leftrightarrow\left\{\operatorname { S e q } ( z ) \& ( \exists m < K ( z ) ) \left[m \text { is even \& } P \left(\left\langle(z)_{1}, \cdots,(z)_{m}\right\rangle,\right.\right.\right. \\
\left.\left.\left.\left\langle(z)_{m+1}, \cdots,(z)_{K(z)}\right\rangle\right)\right]\right\} \\
\vee\left\{\operatorname { S e q } ( z ) \& ( \exists m < K ( z ) ) \left[m \text { is odd \& } P \left(\left\langle(z)_{1}, \cdots,(z)_{m}\right\rangle,\right.\right.\right. \\
\left.\left.\left.\left\langle(z)_{m+2}, \cdots,(z)_{K(z)}\right\rangle\right)\right]\right\} .
\end{array}
$$

From these it follows immediately that the class of $\mathfrak{G}_{1}$ relations on an acceptable structure contains all definable relations and is closed under the operations \&, v, $\forall, \exists, \mathcal{S}, \mathbf{S}$. 
2. The main argument. Following $\S 3$ of [7], a relation $R\left(x_{1}, \cdots, x_{n}\right)$ on $A$ is inductively definable iff for some definable $f\left(x_{1}, \cdots, x_{n}\right)$,

$$
R\left(x_{1}, \cdots, x_{n}\right) \Leftrightarrow f\left(x_{1}, \cdots, x_{n}\right) \in \bigcup_{\xi} P^{\xi}
$$

where the sets $P^{\xi}$ are defined inductively $x \in P^{\xi} \Leftrightarrow P\left(x, \bigcup_{\eta<\xi} P^{\eta}\right)$ relative to a given first order formula $P(x, S)$ with only positive occurrences of $S$.

If $R\left(x_{1}, \cdots, x_{n}\right)$ is $\mathbf{G}_{1}$ satisfying (3) with a definable $Q$, it is easy to verify that $R$ satisfies (13) with

$$
\begin{gathered}
P(x, S) \Leftrightarrow(\exists z)(\exists w)[x=\langle z, w\rangle \& \operatorname{Seq}(w) \& K(w)=n \& \text { Seq }(z) \\
\&\left\{Q\left(z,(w)_{1}, \cdots,(w)_{n}\right) \vee[K(z) \text { is even \& }(\forall t)(z *\langle t\rangle \in S)]\right. \\
\vee[K(z) \text { is odd } \&(\exists t)(z *\langle t\rangle \in S)]\}], \\
f\left(x_{1}, \cdots, x_{n}\right)=\left\langle 1,\left\langle x_{1}, \cdots, x_{n}\right\rangle\right\rangle .
\end{gathered}
$$

(Recall that 1 codes the empty sequence.)

To prove the converse, we first notice that each first order $P(x, S)$ with only positive occurrences of $S$ can be put in the form

$$
P(x, S) \Leftrightarrow(\mathbf{Q} z)(\forall y)[D(x, z, y) \Rightarrow y \in S],
$$

where $D(x, z, y)$ is first order and $\mathbf{Q}$ is a finite string of quantifiers. (This can be shown easily by putting $P(x, S)$ in prenex normal form and then putting the matrix in conjunctive normal form.) Now let $P^{\infty}=\bigcup_{\xi} P^{\xi}$ and notice that

$$
x \notin P^{\infty} \Leftrightarrow\left(\mathbf{Q}^{\smile} z\right)(\exists y)\left[D(x, z, y) \& y \notin P^{\infty}\right],
$$

where $\mathbf{Q}^{\smile}$ is the string dual to $\mathbf{Q}$; if we apply the same equivalence to the clause $y \notin P^{\infty}$ within the brackets, we get

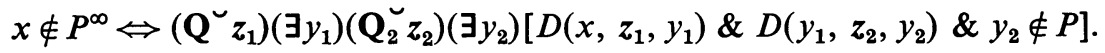

A few more expansions of this form suggest the extrapolation to the infinite

$$
\begin{aligned}
& x \notin P^{\infty} \\
& \Leftrightarrow\left(\mathbf{Q}^{\smile} z_{1}\right)\left(\exists y_{1}\right)\left(\mathbf{Q}^{\smile} z_{2}\right)\left(\exists y_{2}\right) \cdots\left[D\left(x, z, y_{1}\right) \& D\left(y_{1}, z_{2}, y_{2}\right) \& \cdots\right],
\end{aligned}
$$

where $P^{\infty}$ does not occur on the right anymore. A rigorous proof of direction $\Rightarrow$ of (15) is easy. To give a rigorous proof of direction $\Leftarrow$, assume that the right-hand side holds for some $x \in P^{\infty}$ and work for a contradiction. For some $\xi=\xi(x)$ we have $x \in P^{\xi}$ so that

$$
\left(\mathbf{Q}_{z}\right)(\forall y)\left[D(x, z, y) \Rightarrow y \in \bigcup_{\eta<\xi} P^{\eta}\right] ;
$$

this means that in the $\forall-\exists$ finite game that determines the truth of (16), $\exists$ has a winning strategy, call it $\sigma_{x}$. Now let player $\forall$ follow this strategy $\sigma_{x}$ 
against Э's winning strategy in the game determined by the right-hand side of (15). After a finite number of moves we obtain a finite sequence $z_{1}, y_{1}$ such that on the one hand $D\left(x, z_{1}, y_{1}\right)$ and on the other $D\left(x, z_{1}, y_{1}\right) \Rightarrow$ $y_{1} \in \bigcup_{\eta<\xi(x)} P^{\eta}$, so that for some $\eta=\xi\left(y_{1}\right)<\xi(x)$ we have $y_{1} \in P^{\eta}$. We now repeat the argument with $y_{1}$ in the place of $x$ and so on ad infinitum, so that we get an infinite descending chain of ordinals $\xi(x)>$ $\xi\left(y_{1}\right)>\xi\left(y_{2}\right)>\cdots$, which is the desired contradiction.

Taking the negation on the left and the dual on the right of (15), we have

$$
x \in P^{\infty} \Leftrightarrow\left(\mathbf{Q} z_{1} \forall y_{1} \mathbf{Q} z_{2} \forall y_{2} \cdots\right)(\exists k)\left[D\left(x, z_{1}, y_{1}\right) \vee D\left(y_{k-1}, z_{k}, y_{k}\right)\right]
$$

since $\mathbf{Q}$ could have been chosen to be an alternating string beginning and ending with $\exists$, (17) implies almost immediately that $P^{\infty}$ and hence $R$ is $\mathbf{S}_{1}$.

3. Consequences. A set $X$ is universal for a class $\Gamma$ of subsets of $A$ if $X \in \Gamma$ and each set in $\Gamma$ is of the form $\{x:\langle a, x\rangle \in X\}$ for some $a \in A$. Suppose there is a class $\mathcal{T}$ of definable relations on $A$ which contains the relations defined by quantifier free formulas, which contains the relations $x \in N$, Seq $(x), m \leqq n$, which is closed under $\&, \mathrm{v},(\exists m<n)$ and substitution of the functions $\left\langle x_{1}, \cdots, x_{k}\right\rangle,(x)_{i}$ and such that the class of sets (unary relations) in $\mathcal{T}$ has a universal set. The transformations in $\S 1$ then show easily that the class of $\mathbf{G}_{1}$ sets has a universal set. In many cases such classes $\mathcal{T}$ are well known, e.g., for the standard model of second order number theory we can take $\mathfrak{T}=\boldsymbol{\Sigma}_{1}^{0}$. That there is such a $\mathfrak{T}$ for each acceptable structure follows easily from some of the elementary results about prime computability in [5]. Thus for each acceptable $\mathfrak{A}$ there is a universal $\mathbf{S}_{1}$ set, hence a universal inductively definable set. One can now assign ordinals to the elements of this set and then prove a prewellordering theorem like Theorem 7 of [5] using directly an inductive definition (cf. the proof of Lemma 2.6 in [1]). This is sufficient to yield by standard methods most of the basic results about inductively definable relations,

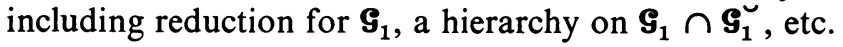

\section{REFERENCES}

1. K. J. Barwise, R. O. Gandy and Y. N. Moschovakis, The next admissible set, J. Symbolic Logic 36 (1.971), 108-120.

2. D. Gale and F. M. Stewart, Infinite games with perfect information, Contributions to the Theory of Games, vol. 2: Ann. of Math. Studies, no. 28, Princeton Univ. Press, Princeton, N.J., 1953, pp. 245-266. MR 14, 999.

3. H. J. Keisler, Finite approximations of infinitely long formulas, Proc. Internat. Sympos. Theory of Models (Berkeley, Calif., 1963), North-Holland, Amsterdam, 1965, pp. 158-169. MR 34 \#2464. 
4. S. C. Kleene, Hierarchies of number-theoretic predicates, Bull. Amer. Math. Soc. 61 (1955), 193-213. MR 17, 4.

5. Y. N. Moschovakis, Abstract first order computability. I, II, Trans. Amer. Math. Soc. 138 (1969), 427-504. MR 39 \#5362.

6. — , Abstract computability and invariant definability, J. Symbolic Logic 34 (1969), 605-633.

7. - The Suslin-Kleene theorem for countable structures, Duke Math. J. 37 (1970), 341-352.

Department of Mathematics, University of California, Los Angeles, CaliFORNIA 90024 